\title{
LA CARIDAD PUNTO DE ENCUENTRO: EL DIÁLOGO ENTRE SABERES PARA POTENCIAR LAS ACTIVIDADES DE PROYECCIÓN SOCIAL
}

\author{
THE CHARITY MEETING POINT: THE DIALOGUE BETWEEN \\ KNOWLEDGE TO ENCOURAGE THE ACTIVITIES OF SOCIAL IMPACT \\ PROJECT
}

Piedad Gañán Rojo ${ }^{1}$

Universidad Pontificia Bolivariana, Colombia ORCID: 0000-0003-2596-2591

https://doi.org/10.21703/2735-6345.2021.22.02.003

Recibido: 28.12.2020

Aceptado: 20.04.2021

\section{Resumen}

En los últimos años las actividades de proyección social se han incrementado. Para adelantarlas es preciso contar tanto con una metodología que permita alcanzar el objetivo propuesto con una visión multidisciplinar de la problemática a la que se espera brindar una solución. Se debe contar para ello con un sentido práctico, pero siempre teniendo presente que las intervenciones se realizan con y en pro del bienestar de otro y evitar que sean consideradas meros indicadores de gestión. Este último aspecto puede ser particularmente complejo cuando las personas con enfoques más técnicos como ingenieros, arquitectos o diseñadores participan o lideran estas acciones. Para conciliar estos puntos y concebir iniciativas que efectivamente logren desarrollo sin detrimento de la condición humana, es posible soportarse en autores que como el Papa Emérito Benedicto XVI reconocen la interrelación entre caridad y técnica. En este trabajo se realiza un análisis de las encíclicas Deus caritas est (DCE) y Caritas in Veritate (CIV) a través del cual se busca identificar claves o elementos que puedan servir de base para ejecutar este tipo de proyectos con una visión más integral del hombre.

Palabras clave: Caridad, proyección social, diálogo de saberes, Deus caritas est (DCE), Caritas in veritate (CIV)

${ }^{1}$ Ingeniero Químico, Doctor en Ingeniería de Materiales. Docente Facultad de Ingeniería Química, Universidad Pontificia Bolivariana (Medellín-Colombia). Correo electrónico: piedad.ganan@upb.edu.co 


\begin{abstract}
In recent years, social impact projects are increasing. These projects require an appropriate methodology that allows for reaching the objective. Define a methodology is more challenging if the project has to be a multidisciplinary vision. When technicians develop these projects, risk can emerge: Focus just on the results and achieve the required indicator. The work of the Emeritus Pope Benedict XVI combines the relevance of the technical aspects to improve human life quality with a deep reflection about the charity. In this study, two of his Encyclical letters, Deus caritas est (DCE) and Caritas in veritate (CIV), are analyzed to identify useful keys for executing social impact projects with an integral human focus. The qualitative research elements were considered for the analysis.
\end{abstract}

Keywords: Charity, social impact project, multidisciplinary dialogue, Deus Caritas Est (DCE), Caritas in veritate (CIV)

\title{
1. Introducción
}

En la actualidad, la mayoría de las entidades públicas o privadas, incluidas las instituciones de educación superior, declaran en sus documentos institucionales que cuentan con departamentos o divisiones a través de las cuales realizan actividades ligadas con la proyección social. En algunos de estos documentos institucionales se pueden encontrar elementos comunes sobre los tipos de acciones que emprenden y que corresponden a la ejecución de proyectos o la prestación de servicios tanto de asesoría, como de consultoría, capacitación o intervención que permitan el mejoramiento de las condiciones de calidad de vida de una comunidad o entorno en particular ${ }^{2}$. Sumado a lo anterior, parte de las acciones de proyección social guardan relación con las indicaciones de la Guía de responsabilidad social ISO 26000:2010³, según la cual una organización debe ser responsable "ante los impactos que sus decisiones y actividades ocasionan en la sociedad y el medio ambiente" y además debe tener un "comportamiento

${ }^{2}$ Esta idea fue establecida tras la revisión de algunas de las páginas web de entidades educativas, principalmente universidades latinoamericanas, que en sus documentos institucionales disponibles en sus portales web indican lo que es para cada una de ellas las acciones de proyección social. Ejemplo de ello corresponde a: Corporación Universitaria Uniminuto-Colombia.

(http://www.uniminuto.edu/web/llanos/proyeccion-social, citado 22 enero 2021). Adicionalmente, la Asociación Colombiana de Universidades (ASCUN) realizó un estudio sobre cómo es la proyección social que realizan las universidades de este país que se puede encontrar en el vínculo: https://ascun.org.co/noticias/detalle/ ascun-presenta-resultados-proyeccion-social-universitaria, citado 22 enero 2021.

${ }^{3}$ ISO, Guía de responsabilidad social ISO 26000:2010, Iso, Ginebra 2010, 1-5. En adelante, ISO 26000:2010. 
ético y transparente"4. Este tipo de intervenciones en muchos sentidos podrían tener como propósito contribuir a alcanzar lo que se menciona en el apartado 6 de la encíclica Populorum progressio de San Pablo VI sobre las aspiraciones del hombre "Verse libres de la miseria, hallar con más seguridad la propia subsistencia, la salud, una ocupación estable”.

Al momento de concebir, diseñar o ejecutar este tipo de proyectos puede caerse en el riesgo de buscar el logro, el cumplimiento completo del objetivo principal, a expensas por ejemplo de los componentes culturales, de tal forma que al final no se alcance a potenciar lo humano, sino que, muy por el contrario, este se considere un mero instrumento y los resultados del proyecto solo se conviertan en estadísticas o reportes que se consolidan en un informe. Esto se puede observar en fallos cometidos en proyectos de cooperación ejecutados en diversas regiones del mundo que al excluir la participación de las comunidades o dejar que intervengan solo al final, más como receptores de la ayuda que como actores fundamentales, dejaron de ser viables una vez que la intervención concluye. Otros elementos que se pueden sumar a las dificultades de las acciones de proyección social están ligados a los enfoques que se adoptan, por ejemplo, cuando tienen matices con una marcada visión técnica pueden tener a excluir o minimizar el valor de la social, o en el sentido opuesto un enfoque más social tiene a excluir o relegar el técnico. De este último aspecto puede surgir una nueva dificultad y es que las personas más ligadas a las áreas técnicas pueden tender a auto marginarse o preferir, en lugar de utilizar su potencial y capacidad, orientar sus formas de actuar hacia otras áreas o incluso excluirse completamente y dedicarse a ser simples donantes pasivos que no se involucran. La pérdida que aquí ocurre no solo es de la posibilidad de disponer de múltiples miradas para reconocer la realidad, de por sí muy valiosa, sino además de los ya probados beneficios que sobre la salud humana tiene participar en estas acciones tal como diferentes estudios neurocientíficos indican, el ejercicio de acciones de caridad genera efectos positivos sobre la bioquímica del cuerpo ${ }^{6}$.

4 ISO 26000:2010.

5 Pablo VI, Carta Encíclica Populorum progressio, n. 2, disponible en: http://www.vatican.va/content/paul-vi/es/encyclicals/documents/hf_p-vi_ enc_26031967_populorum.html, citado 27 enero 2021.

6 Cf. N. LAURENDEAU, "Christian mysticism and science. The psychological dimension", Theology and Science 11/1 (2013), 24-26. 
Por otro lado, se puede considerar que las actividades de proyección social pueden guardar relación con la caridad, pues si se considera al menos bajo una de las definiciones de la $R A E$, caridad es la "actitud solidaria con el sufrimiento ajeno", justo lo permita superar las causas que le generan el sufrimiento actual. Como lo menciona R. Muñoz, han existo diferentes revisiones desde múltiples ópticas sobre el tema de la caridad y la mayoría de ellas la ven como algo que debe ser suprimido, porque en lugar de liberar al hombre lo esclaviza ${ }^{8}$.

Al revisar ambos planos de intervención, el con orientación más humano o el más técnico, es necesario entonces continuar en la construcción de una visión más multidisciplinar y por ello surge la pregunta ¿̇cómo poder potenciar las experiencias que en diseño, producción, ejecución y evaluación de proyectos tienen muchas profesionales de áreas técnicas, como ingenieros, diseñadores industriales o arquitectos, de tal forma que las actividades de proyección social puedan ser un verdadero aporte y generen bienestar no solo en las poblaciones directamente beneficiadas sino a todas las personas involucradas en la cadena y que incluyen personas de múltiples profesiones así como a los benefactores que realizan sus aportes bien sea en dinero o en especie?. Para abordar este tema, es necesario considerar pensadores en los cuales lo humano y lo científico-técnico sean complementarios y uno de los mejores exponentes de este sentido es el Papa emérito Benedicto XVI, quien tanto previamente como a lo largo de su pontificado dio claras indicaciones sobre la importancia del enfoque interdisciplinario y de lo que aporta al analizar realidades complejas ${ }^{9}$, como se evidencia cuando indica en su encíclica Caritas in Veritate que "solo con la caridad, iluminada por la luz de la razón y de la fe, es posible conseguir objetivos de desarrollo con un carácter más humano y humanizador" ${ }^{10}$. En el presente trabajo realiza un análisis básico de sus encíclicas Deus caritas est y Caritas in Veritate,

${ }^{7}$ Diccionario de la Real Academia de la Lengua Española, RAE, consultado el 20 de enero de 2021.

${ }^{8}$ Cf. R. MUÑOZ, "Caritas. Amor cristiano y acción social”, Scripta Theologica 38/3 (2006) 1005-1007.

9 Cf. M. SÁNCHEZ Sorondo, Ex aedibvs academicis in civitate vaticana, Editrice Vaticana, Roma 2015, 10.

${ }^{10}$ BENEDICTO XVI, Carta encíclica Caritas in veritate, n. 8, en: http://www.vatican. $\mathrm{va} /$ content/benedict-xvi/es/encyclicals/documents/hf_ben-xvi_enc_20090629_ caritas-in-veritate.htmll, citado 27 enero 2021. (En adelante: CIV). 
donde él aborda la caridad como eje central. Se buscan identificar aspectos que puedan ser de utilidad al momento de diseñar y realizar actividades de proyección social, que consideren una mirada más integral del ser humano, pues tanto la caridad como la técnica son expresiones profundamente humanas y a través de las cuales pueda ser posible establecer puentes de comunicación para este tan necesario diálogo entre saberes

\section{Consideraciones básicas sobre las encíclicas}

La encíclica Deus caritas est fue presentada el 25 de diciembre del 2005 y es la primera de las tres encíclicas de su pontificado. Tal como lo indica el Papa en la introducción está conformada por dos partes: La primera de ellas tal como se indica en el apartado 1 tiene un "carácter más especulativo" pues desea analizar "algunos puntos esenciales sobre el amor de Dios" ${ }^{11}$. El comienzo de esta primera parte tiene un carácter claro y magistral, define los términos a usar incluso su origen etimológico y progresivamente va indicando al lector su visión del tema. La forma en que se encuentra divida esta sección permite ver progresivamente la presentación de la temática de tal forma que es posible comprender su conclusión en la que da una noción de unicidad cuando dice "El amor crece a través del amor...a Dios nos une y, mediante este proceso unificador, nos transforma en un Nosotros, que supera nuestras divisiones y nos convierte en una sola $\operatorname{cosa}{ }^{12}$. Establecidas las bases, en la segunda parte busca suministrar una "índole más concreta" para cumplir con este mandamiento del amor que define en la primera parte.

La encíclica Caritas in veritate, del 29 de junio del 2009, cuenta con más elementos prácticos que Deus caritas est, pues se encuentra orientada a un mundo, que no hace mucho, había experimentado la crisis económica del año 2008. Este texto, tal como se indica en el apartado 8, busca rendir un homenaje a la publicación de otro importante texto, la encíclica de Papa Pablo VI Populorum Progressio, que fue proclamada el 26 de marzo de 1967. Nuevamente encontramos la claridad en la definición de los términos

${ }^{11}$ Benedicto XVI, Carta encíclica Deus caritas est, n. 18, disponible en: http:// www.vatican.va/content/benedict-xvi/es/encyclicals/documents/hf_ben-xvi_ enc_20051225_deus-caritas-est.html, citado 27 enero 2021. (En adelante: DCE).

${ }^{12}$ DCE 13. 
$\mathrm{y}$ aspectos que desean resaltar y sobre los cuales se lanzan las propuestas allí consignadas.

Desde su publicación ambas encíclicas han sido sometidas a profundos análisis pues cuentan con una gran riqueza para variados públicos. Algunos de dichos autores como Múnera ${ }^{13}$ analizan estos textos en clave de la doctrina social de la Iglesia y enfatiza en los elementos relacionados con la justicia y la actuación de la Iglesia Católica; por su parte Monseñor Paul Cordes realiza un análisis más teológico a la primera de ellas, cuando por ejemplo indica que la afirmación central de Deus caritas est es "el hecho de que toda caridad entre los seres humanos está enraizada teocéntricamente, esto es, tenga su fuente en Dios" ${ }^{14}$. Por su parte, J. Sapena y otros las toman como documentos de apoyo en su análisis sobre la pobreza, el desarrollo y la sostenibilidad para indagar sobre aspectos que, como los reducidos ingresos, el papel de las instituciones y la pérdida de valores tienen influencia sobre lo complejo que es para algunas comunidades el escapar de la llamada trampa de la pobreza ${ }^{15}$. Por otro lado, Frémeaux y otros consideran estos textos para una reflexión sobre el papel de la tecnociencia y la economía ${ }^{16}$. A su vez Vaccaro y Sison concentran su estudio en Caritas in veritate para realizar un análisis sobre la ética en los negocios, la responsabilidad social y la transparencia en diferentes organizaciones ${ }^{17}$. A lo anterior es posible sumar el uso que realiza de estos textos Luque para abordar el tema de la educación, en especial la educación del espíritu tanto en contextos religiosos como seculares ${ }^{18}$.

Estos ejemplos sobre el uso de las encíclicas para abordar temas tan diversos ejemplifican que el pensamiento del Papa Benedicto XVI es integra-

${ }^{13}$ D. Múnera, Curso de doctrina social de la Iglesia: humanismo cristiano y desarrollo humano, Industria Gráfica Litoservicios, Medellín 2012, vol. 2, 250.

${ }^{14}$ J. CoRDES, "Deus caritas est. Sobre la primera encíclica del Papa Benedicto XVI", Scripta Theologica 38/3 (2006) 972.

${ }_{15}$ J. SAPENA, ET AL., "Some reflections on poverty eradication, true development and sustainability within CST", Journal of Innovation \& Knowledge 3/2 (2018) 90.

${ }_{16}$ Cf. S. FrémeAux - F. R. PUYOU - G. Michelson, "Beyond accountants as technocrats: A common good perspective", Critical Perspectives on Accounting 6768 (2020) 8.

${ }^{17}$ Cf. A. VACCARo - A. J. G. Sison, "Transparency in business: The Perspective of Catholic Social Teaching and the "Caritas in Veritate", Journal of Business Ethics 100/1 (2011) 19-20.

${ }^{18}$ Cf. D. LuQUE, "La educación del espíritu. Líneas de pensamiento para una teoría de la educación", Edetania 57 (2020) 39-43. 
dor, multidisciplinar y que al realizar aproximaciones entre áreas como la teología, la filosofía, la tecnología y la ciencia le permiten al lector acercarse a esferas de conocimiento que le son ajenas, pero que en lugar de alentar la separación, invita de una forma magistral a que persevere y encuentre elementos de valor para su propia búsqueda, para su propia reflexión.

\section{Metodología}

Para realizar este análisis se tomaron elementos de la investigación cualitativa. Para ello las dos encíclicas fueron analizadas inicialmente de forma separada considerando aislar unidades de texto. Tras su repetida lectura, se procedió con la codificación y cada grupo de códigos fue agrupado en subcategorías que a su vez fueron ordenadas en categorías y éstas fueron relacionadas con parte de los corpus teóricos ligados a las acciones comunes, similares o complementarias que deben ser consideradas tanto en la conceptualización como en la realización de proyectos de diseño, ingeniería e innovación. El sistema de categorías construido busca cumplir con las características de ser exhaustivo, cubrir unidades diferencias de información, que exista exclusión mutua y que cada unidad se incluye en una sola categoría. Además, que las categorías sean relevantes tanto respecto a la pregunta de investigación realizada como al contenido analizado. ${ }^{19}$ Para la identificación de las acciones comunes, similares o complementarias que tienen los diferentes tipos de proyectos se consideraron variadas fuentes bibliográficas que incluyen: Para los proyectos de diseño las consideraciones en Pugh, Ulrich y Eppinger ${ }^{20}$; para los de proyectos de ingeniería García Melón y otros, Glorioso y Cross, y en Fargnoli y Sako ${ }^{21}$; en cuan-

19 Cf. G. Rodríguez - J. GIL- E. GARcía, Metodología de la investigación cualitativa, Aljibe, Málaga 1996, 200; S. Osses BustingorRY- I. SÁNCHEz TAPIA- F. M. IBÁÑEZ MANSILLA, "Inverstigación cualitativa en educación. Hacia la generación de teoría a través del proceso analítico”, Estudios pedagógicos 32/1 (2006) 123-124.

${ }_{20} \mathrm{Cf}$. S. Pugh, Total design: integrated methods for successful product engineering, Prentice Hall, Harlow 1991, 278; K. Ulrich- S. EPPINGER, Product design and development, McGraw-Hill, Nueva York 2012, 368.

${ }^{21}$ Cf. M. GARCía Melón, ET AL., Fundamentos del diseño en la ingeniería, Limusa, México 2010, 380; R. GLorioso, Introduction to engineering, Prentice Hall, New York 1974, 461; N. CROss, Engineering design methods: Strategies for product design, Wiley, New York 2020, 230; M. FARGNOLI- T. SAKO, "Uncovering differences and similarities among quality function deployment-based methods in Design for X: Benchmarking in different domains", Journal Quality Engineering 29/4 (2017) 690712 . 
to a la innovación se consideraron autores como Pérez Breva y Salame ${ }^{22}$.

Las acciones comunes, similares o complementarias que se identificaron en las diferentes metodologías para realizar proyectos corresponde a: a) Identificar una necesidad considerando aspectos como los tomados de la innovación relacionados con un análisis de contexto que permite generar o conceptualizar un proyecto; b) generar especificaciones preliminares que permitan brindar una solución a la problemática planteada, generar y analizar alternativas - generar los prototipos una vez ha sido analizadas las viabilidades técnicas, económicas, éticas, normativas y ambientales; c) ejecución del proyecto.

\section{Resultados y análisis}

En las dos encíclicas analizadas el Papa Emérito busca instar a los feligreses a ser metódicos al momento de considerar la realización de una actividad de caridad, esto implica tener en consideración aspectos como lo indica de "una orientación decisiva" ${ }^{23}$, y por ello es importante contar con una metodología que oriente la acción. Como se mencionaba en otro momento del presente texto, las encíclicas comienzan indicando claramente su propósito, su intencionalidad, la estructura que tienen, lo que allí encontrará el lector además de definir los términos centrales de tal forma que no se presten a significados o interpretaciones que puedan generar ambigüedad. Con esto también busca homologar los conceptos que se trabajan y establecer distancia con interpretaciones o precisiones de otros autores. De forma adicional en ambas es posible resaltar el profundo conocimiento que sobre el tema de la caridad y de la relación entre la caridad y la verdad tiene el Papa, pues la extensa bibliografía que plantea, su revisión y la forma en que se encuentran estructurados los textos facilitan su lectura y comprensión. Incluso en el caso de DCE es posible encontrar el análisis de posiciones contrarias a los postulados defendidos por el autor.

${ }^{22}$ L. PÉREZ Breva, Innovating: a doer's manifesto for starting from a hunch, prototyping problems, scaling up, and learning to be productively wrong, The MIT Press, Cambridge 2018, 424. Cf. H. A. SALAME, La planeación y el desarrollo de productos, Universidad Piloto de Colombia, Bogotá 2013, 219. 222.

23 BenEDICTO XVI, Fe y ciencia: un diálogo necesario, Sal Terrae, Santander 2011, 
Pese a que ambos documentos son fundamentales dentro de la doctrina social de la Iglesia católica ${ }^{24}$ independientemente de los aspectos confesionales, en algunos apartados son altamente orientadores respecto a la importancia que se deben relacionar las acciones de caridad y la ciencia y la importancia del conocimiento multidisciplinar, que se evidencian por ejemplo cuando se menciona en $C I V$ que "el desarrollo humano integral como vocación exige también que se respete la verdad" o cuando indica que "Al afrontar los fenómenos que tenemos delante, la caridad en la verdad exige ante todo conocer y entender, conscientes y respetuosos de la competencia específica de cada ámbito del saber" ${ }^{25}$.

En un análisis más detallado sobre este papel interdisciplinar que puede orientar las acciones de caridad o de proyección social, según la definición que se tome, se puede resaltar lo que se indica en CIV cuando se expresa que:

“... el tema del desarrollo humano integral adquiere un alcance aún más complejo: la correlación entre sus múltiples elementos exige un esfuerzo para que los diferentes ámbitos del saber humano sean interactivos, con vistas a la promoción de un verdadero desarrollo de los pueblos. Con frecuencia, se cree que basta aplicar el desarrollo o las medidas socioeconómicas correspondientes mediante una actuación común. Sin embargo, este actuar común necesita ser orientado" ${ }^{26}$.

En las dos encíclicas se reconoce la importancia y el valor que los conocimientos técnicos tienen en el progreso del hombre, además de su impronta en el carácter profundamente humano de los mismos, se insiste a tal punto que en $C I V$ en el apartado 69 se indica que:

"La técnica es el aspecto objetivo del actuar humano... Por eso, la técnica nunca es sólo técnica. Manifiesta quién es el hombre y cuáles son sus aspiraciones de desarrollo, expresa la tensión del ánimo humano hacia la superación gradual de ciertos condicionamientos materiales" ${ }^{27}$.

Es entonces necesario que en todas las acciones que se enfocan en el mejoramiento de la calidad de vida, como es justo lo que buscan los de

${ }^{24}$ Cf. D. MÚNERA, Curso de doctrina social..., 200.

${ }_{25}^{25}$ CIV 18, 30.

${ }^{26}$ CIV 30.

${ }^{27}$ CIV 69. 
proyección social, exista una visión multidisciplinar en donde la técnica y la ciencia no se encuentren desconectadas, que tengan una relación armónica con lo humano, a través de la cual sea posible establecer un diálogo respetuoso y permanente y que propugne por "en la común responsabilidad respecto al recto uso de la razón” ${ }^{28}$ y que permita poner el conocimiento técnico y científico al servicio del bienestar humano ${ }^{29}$. Este diálogo, tal como se indica en $C I V$ debe ser "un diálogo que, para ser eficaz, ha de tener como punto de partida una toma de conciencia de la identidad específica de los diversos interlocutores" 30 .

Con este reconocimiento de la importancia que tiene la interdisciplinariedad para el desarrollo de cualquier proyecto a continuación se presenta el análisis de las encíclicas considerando siempre identificar elementos que puedas ser de utilidad para apoyar la realización de las actividades de proyección social. Las categorías y subcategorías identificadas de la lectura de las dos encíclicas seleccionadas se presentan en la tabla 1, mientras que en la tabla 2 se realiza una relación con algunas de las acciones comunes, similares o complementarias que tienen los proyectos de investigación, ingeniería, diseño e innovación, previamente identificadas, buscando establecer efectivamente este punto de unión entre la caridad y las etapas para la realización de estos.

La primera categoría, indicada en la tabla 1, corresponde a los múltiples elementos que pueden definir, caracterizar y abarcar la caridad, se agruparon como elementos de la caridad. Al ser textos con elementos y bases teológicos que hacen parte de la doctrina social de la Iglesia es necesario considerarlos como bases para la caridad y así lo hace evidente el Papa cuando en CIV indica que "la caridad es la vía maestra de la doctrina social de la Iglesia" ${ }^{31}$. Además de esto es posible identificar que la caridad está vinculada a otros elementos centrales como es el ser una expresión de auténtica humanidad y que por demás debe tener muy claramente estableci-

${ }^{28}$ BENEDICTO XVI, Fe y ciencia..., 50.

${ }^{29}$ Cf. P. GaÑán RoJo, "En el ejercicio de la caridad confluyen tecnología, ingeniería y humanismo", Revista Universidad Pontificia Bolivariana 58/158 (2019) 42-43; P. GAÑÁN ROJO "Responsabilidad individual y compromiso ambiental en los tiempos del COVID-19", Revista Universidad Pontificia Bolivariana 59/159 (2020) 130.

${ }^{30}$ CIV 26.

${ }^{31}$ CIV 2. 
do su objetivo de tal forma que no se convierta como indica el Papa en una actividad de asistencia social. Un elemento clave para la realización de todo proyecto, tal como se menciona en la tabla 2, es definir o acotar el problema que se desea resolver o abordar. En relación con esto, es posible encontrar explícitamente esta indicación en las encíclicas cuando se menciona que es preciso saber que las acciones realizadas deben dar respuesta a una necesidad y esto se manifiesta en DCE en varias partes y directamente donde indica que "la caridad cristiana es ante todo y simplemente la respuesta a una necesidad inmediata en una determinada situación" ${ }^{22}$. Otros elementos relevantes, que son posibles considerar, tanto en la concepción en las acciones de caridad, como los de proyección social y que se resumen en la tabla 1, son: la independencia de ideologías pues "el compromiso por el bien común, cuando está inspirado por la caridad, tiene una valencia superior al compromiso meramente secular y político"33; el reconocimiento que, pese a realizarlas, siempre serán necesarias de continuar llevándose a cabo, pues bien, se indica "nunca habrá situaciones en las que no haga falta la caridad" 34 .

En estas encíclicas se resalta el sentido práctico que se revela en ambas, y muy al comienzo cuando en Deus caritas est en su introducción indica que en la segunda parte del texto busca hacerlo de una forma más práctica cuando menciona que "tendrá una índole más concreta, pues tratará de cómo cumplir de manera eclesial el mandamiento del amor al prójimo" y tal como lo menciona también allí el Papa Emérito su propósito es "insistir sobre algunos elementos fundamentales, para suscitar en el mundo un renovado dinamismo de compromiso en la respuesta humana al amor divino." ${ }^{35}$. Con esta base y siguiendo con la segunda categoría de la tabla 1 que corresponde a la relación entre la caridad y verdad es posible, tal como se indica en la tabla 2, relacionar con la etapa de concepción de los proyectos, pues en este punto es fundamental considerar las opiniones de otros, incluso cuando algunas de ellas puedan ser contrarias en especial porque muchas visiones permiten establecer una más completa de la realidad y a su vez facilitan el delimitar el problema a abordar.

\footnotetext{
${ }^{32}$ DCE 31.

${ }_{33} \mathrm{CIV} 7$.

34 DCE 29.

35 DCE 1.
} 
Tabla 1. Resultados del análisis del texto de Caritas in veritate y Deus caritas est

\begin{tabular}{|c|c|}
\hline CATEGORÍAS & SUBCATEGORÍAS \\
\hline \multirow{14}{*}{ Elementos de la caridad } & Doctrina social \\
\hline & Ágape \\
\hline & Dios es caridad \\
\hline & Expresión de auténtica humanidad \\
\hline & Caridad es amor \\
\hline & Caridad como don \\
\hline & No es una actividad de asistencia social \\
\hline & Desarrollo humano como vocación \\
\hline & $\begin{array}{l}\text { Elemento fundamental en las relaciones } \\
\text { humanas }\end{array}$ \\
\hline & Actividad ordenada \\
\hline & Siempre necesaria \\
\hline & Debe ser ejercida de forma respetuosa \\
\hline & Libre de ideologías \\
\hline & Respuesta a una necesidad \\
\hline \multirow{3}{*}{ Relación entre caridad y verdad } & Verdad que libera la caridad \\
\hline & $\begin{array}{l}\text { Valoración entre caridad y verdad (ra- } \\
\text { zón) }\end{array}$ \\
\hline & Interacción entre ambas \\
\hline \multirow{8}{*}{$\begin{array}{l}\text { Riesgos que enfrenta la caridad sin ver- } \\
\text { dad }\end{array}$} & Caridad mal entendida \\
\hline & Pérdida de contexto \\
\hline & Irrelevancia \\
\hline & Fidelidad a la verdad \\
\hline & Caer en mero sentimentalismo \\
\hline & Ser excluida \\
\hline & Perder su rumbo u orientación \\
\hline & Convertirse en una farsa \\
\hline
\end{tabular}




\begin{tabular}{|c|c|}
\hline \multirow{7}{*}{$\begin{array}{l}\text { Riesgos que enfrenta la razón sin la ca- } \\
\text { ridad }\end{array}$} & $\begin{array}{l}\text { Solo establecer relaciones de convenien- } \\
\text { cia }\end{array}$ \\
\hline & Ilusión de omnipotencia \\
\hline & Visión empirista y escéptica de la vida \\
\hline & $\begin{array}{l}\text { Posición ambivalente: Omnipotente o } \\
\text { antihumana }\end{array}$ \\
\hline & $\begin{array}{l}\text { Insuficiente para resolver los problemas } \\
\text { del hombre }\end{array}$ \\
\hline & Sectorización del saber \\
\hline & Incertidumbre sobre las consecuencias \\
\hline \multirow{8}{*}{ Concepción de la acción o solución } & Acción ética y responsable \\
\hline & Conciencia sobre el bien común \\
\hline & Respeto por las personas y los pueblos \\
\hline & Ser flexibles \\
\hline & $\begin{array}{l}\text { Involucrar a los beneficiarios desde la } \\
\text { planificación }\end{array}$ \\
\hline & $\begin{array}{l}\text { Considerar los impactos en las genera- } \\
\text { ciones sucesivas }\end{array}$ \\
\hline & $\begin{array}{l}\text { Considerar involucrar todos los actores } \\
\text { posibles }\end{array}$ \\
\hline & $\begin{array}{l}\text { Contar con los recursos necesarios para } \\
\text { su ejecución }\end{array}$ \\
\hline \multirow{4}{*}{ Desarrollo del proyecto o de la acción } & $\begin{array}{l}\text { Aplicar criterios de seguimiento y acom- } \\
\text { pañamiento }\end{array}$ \\
\hline & $\begin{array}{l}\text { Humanismo cristiano clave para el desa- } \\
\text { rrollo de un proyecto }\end{array}$ \\
\hline & $\begin{array}{l}\text { Transparencia en la acción - información } \\
\text { oportuna sobre los avances }\end{array}$ \\
\hline & $\begin{array}{l}\text { Cualidades de las personas que realizan o } \\
\text { ejecutan la actividad }\end{array}$ \\
\hline
\end{tabular}


Tabla 2. Relación entre los conceptos vinculados a proyectos con las categorías identificadas en las encíclicas

\begin{tabular}{|c|c|c|}
\hline $\begin{array}{l}\text { Acciones comunes, si- } \\
\text { milares o complemen- } \\
\text { tarias de proyectos de } \\
\text { diseño, investigación o } \\
\text { innovación }\end{array}$ & Definición & $\begin{array}{l}\text { Categorías o subcategorías } \\
\text { identificas en la lectura de } \\
\text { las encíclicas }\end{array}$ \\
\hline $\begin{array}{l}\text { Identificar una nece- } \\
\text { sidad -análisis de con- } \\
\text { texto - conceptualiza- } \\
\text { ción de un proyecto }\end{array}$ & $\begin{array}{l}\text { Fases ligadas a establecer } \\
\text { el objetivo general de una } \\
\text { actividad y que brinde la } \\
\text { respuesta más adecuada } \\
\text { a una problemática clara- } \\
\text { mente definida y delimi- } \\
\text { tada }\end{array}$ & 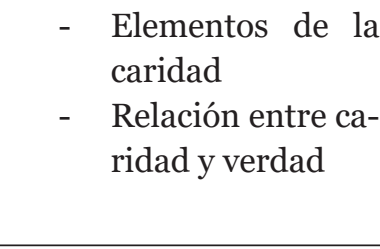 \\
\hline $\begin{array}{l}\text { Generar especificacio- } \\
\text { nes preliminares que } \\
\text { permitan brindar una } \\
\text { solución a la problemá- } \\
\text { tica planteada, generar } \\
\text { y analizar alternativas } \\
\text { - generar los proto- } \\
\text { tipos una vez ha sido } \\
\text { analizadas las viabili- } \\
\text { dades técnicas, econó- } \\
\text { micas, éticas, normati- } \\
\text { vas y ambientales }\end{array}$ & $\begin{array}{l}\text { Con la claridad del proble- } \\
\text { ma es posible comenzar } \\
\text { un proceso, que puede ser } \\
\text { iterativo donde se analizan } \\
\text { diferentes alternativas de } \\
\text { solución. } \\
\text { Las acciones principales } \\
\text { pueden ser subdivididas } \\
\text { en tareas con el fin de que } \\
\text { cada una de ellas permita } \\
\text { cumplir con el cumpli- } \\
\text { miento del objetivo central } \\
\text { Son procesos que requie- } \\
\text { ren de una observación, } \\
\text { planeación y análisis para } \\
\text { establecer las alternativas } \\
\text { más viables } \\
\text { Riesgos de estos procesos: } \\
\text { alejarse de los objetivos } \\
\text { planteados }\end{array}$ & 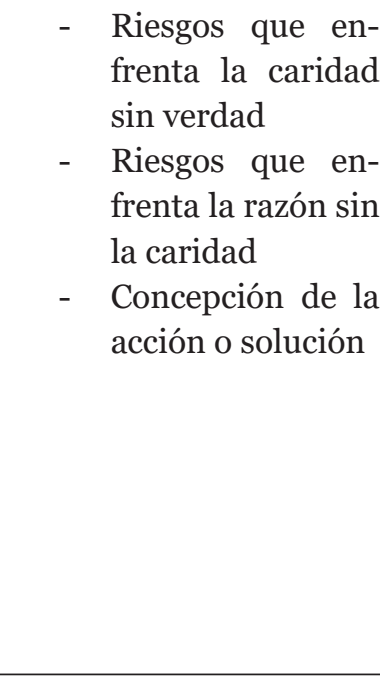 \\
\hline Ejecución del proyecto & $\begin{array}{l}\text { Una vez establecidas clara- } \\
\text { mente las acciones a reali- } \\
\text { zar, la forma de llevarlas y } \\
\text { los sistemas de monitoreo } \\
\text { y control se procede con su } \\
\text { ejecución. }\end{array}$ & $\begin{array}{l}\text { - Desarrollo del } \\
\text { proyecto o de la } \\
\text { acción }\end{array}$ \\
\hline
\end{tabular}

En este sentido la verdad libera la caridad entre otros aspectos cuando en CIV se indica que "La verdad libera a la caridad de la estrechez de una emotividad que la priva de contenidos relacionales y sociales, así como de 
un fideísmo que mutila su horizonte humano y universal" ${ }^{36}$. El éxito entonces de una acción debe estar mediado por la relación entre ambos componentes, de tal forma que como se indica en CIV:

"La clave del desarrollo está en una inteligencia capaz de entender la técnica y de captar el significado plenamente humano del quehacer del hombre, según el horizonte de sentido de la persona considerada en la globalidad de su ser" ${ }^{37}$.

Una vez que se tiene definido el problema y se han establecido los objetivos o las preguntas base, y según la metodología respectiva que se siga en cada proyecto, una siguiente fase común en todos, como se indica en la tabla 2, se encuentra asociada con el generar especificaciones preliminares, esto puede implicar la generación de una serie de propuestas de solución que deben ser analizadas en función de su viabilidad técnica, económica, ética, normativa y ambiental. Para apoyar este aspecto, es preciso considerar una serie de alertas que permiten que las soluciones finalmente aceptadas puedan ser potencialmente implementadas o ejecutadas y que no se desvíen de su objetivo. Estas alertas cobren tanto los riesgos a los que se enfrenta la acción de caridad o de proyección social sin el apoyo de la razón como los que enfrenta la razón sin la caridad. En los textos es posible encontrar claves para tener en cuenta no solo en esta fase de desarrollo de los proyectos, sino en otros momentos como en su ejecución. En cuanto a los riesgos que enfrenta la caridad sin verdad están no solo los ligados a que pueda caer en interpretaciones indebidas, sino que pierda su sentido o el contexto bajo el cual la acción deba ser realizada a tal punto que se torne irrelevante. En toda acción de proyección social, tal como lo ratifica el Papa, para las acciones de caridad es de vital importancia evitar caer en el mero sentimentalismo. Esto se hace más concreto en $D C E$ donde se indica: “... una fuerza purificadora para la razón misma. (...) La libera de su ceguera y la ayuda así a ser mejor ella misma. La fe permite a la razón desempeñar del mejor modo su cometido y ver más claramente lo que le es propio" 38 .

Por otra parte, dejar de lado los elementos de caridad y formular y

${ }^{36}$ DCE 3.

${ }_{37}$ CIV 70.

${ }^{38}$ DCE 28. 
orientar las soluciones solo considerando aspectos netamente técnicos "no se asegura sólo con el progreso técnico" 39 pues este puede tener graves riesgos que se enumeran en la tabla 1 y entre los que se pueden resaltar: que las acciones emprendidas solo tiene valor o son importantes en la medida en que se puedan establecer relaciones de conveniencia; también la alerta sobre caer en el peligro que la técnica y la ciencia resuelven todos los problemas cubriendo así la razón de una ilusión de omnipotencia, que a su vez puede generar una visión más escéptica de la vida. La técnica tal como se ha mencionado en otros apartados del presente análisis, es considerada con un componente altamente humano "vinculado a la autonomía y libertad del hombre" que lo atrae fuertemente "porque lo rescata de las limitaciones físicas y le amplía el horizonte ${ }^{40}$, además de lo expresado en CIV:

"la técnica permite dominar la materia, reducir los riesgos, ahorrar esfuerzos, mejorar las condiciones de vida. Responde a la misma vocación del trabajo humano: en la técnica, vista como una obra del propio talento, el hombre se reconoce a sí mismo y realiza su propia humanidad"41.

Pero esta misma técnica también podría adoptar una posición ambivalente, por un lado, como se mencionaba antes, tener la visión que todo lo puede y por el otro alejarse del hombre, incluso hasta llegar a ser considerada como algo antihumano, punto que se viene discutiendo por varios autores como Gómez sobre las implicaciones de la tecnociencia ${ }^{42}$. Es de vital importancia continuar insistiendo que al analizar sobre cuáles deben ser las soluciones más apropiadas para implementar con el fin de resolver o contribuir a la problemática previamente planteada se tenga conciencia sobre la incertidumbre que pueda existir sobre las consecuencias de su aplicación.

Una vez considerados los riesgos, es necesario que la solución concebida y que se espera implementar sea ética, cumpla con las condiciones de aportar al bien común, no afecte a los implicados y que durante este proceso de definición fuera tan analizada y meditada con la participación de

${ }^{39}$ CIV 8.

${ }^{40}$ CIV 70.

${ }^{41}$ CIV 69.

42 V. GómEz, La técnica: El umbral entre la cultura material y el materialismo de consumo. Por un debate social sobre la tecnociencia, Universidad Pontificia Bolivariana, Medellín 2020, 114. 
todos los agentes posibles. Involucrar a los beneficiarios desde la planificación es indispensable, porque como se indica en $C I V$ "Las soluciones se han de ajustar a la vida de los pueblos y de las personas concretas, basándose en una valoración prudencial de cada situación". Además "porque no hay recetas universalmente válidas" 43 .

Dentro de los elementos también clave de tener presente en la selección de esta acción es considerar la disposición de contar con todos los recursos necesarios para realizarla.

Una vez que se ha seleccionado la acción a realizar y comience su implementación o ejecución, de las encíclicas es posible obtener nuevas guías en este sentido. En este caso en la tabla 1 se encuentran en la categoría desarrollo del proyecto o de la acción. Dentro de los aspectos a considerar están la ratificación de la importancia de la transparencia en la acción que puede llevarse a cabo mediante una entrega de información oportuna sobre los avances de los proyectos. Otros aspectos por resaltar es el ligado a las cualidades de las personas que participan en la ejecución de los proyectos quienes debe ser competentes y además de contar con cualidades humanas pues como se indica “... los seres humanos necesitan siempre algo más que una atención sólo técnicamente correcta"44. Esto implica que es preciso evitar adoptar posiciones de superioridad, ser capaces de reconocer también ellos serán beneficiados:

"Quien es capaz de ayudar reconoce que, precisamente de este modo, también él es ayudado; el poder ayudar no es mérito suyo ni motivo de orgullo. Esto es gracia"45.

Ya el Papa con esta exhortación se adelantaba a indicar los beneficios que para la salud humana supone el ayudar a otros, tal como lo viene estudiando y comprobando la neurociencia. ${ }^{46}$ Estas personas realizarán con humildad su actividad evitando que en la medida de lo posible esto las desborde, a tal punto que pueda afectar su integridad. Sumado a lo anterior, también estas personas deben contar con las competencias técnicas reque-

${ }^{43}$ CIV 47.

${ }_{44}$ DCE 31.

${ }^{45}$ DCE 35.

46 Cf. N. Laurendeau, "Christian mysticism and science..."; M. JakovlJEvic, "Psychiatry and religion: Opponents or collaborators - The power of spirituality in contemporary psychiatry", Psychiatria Danubina 29/1 (2017) 82-88. 
ridas, pues tal como se indica en DCE:

“... es preciso que sean competentes profesionalmente: quienes prestan ayuda han de ser formados de manera que sepan hacer lo más apropiado y de la manera más adecuada, asumiendo el compromiso de que se continúe después las atenciones necesarias" ${ }^{47}$.

Además, de siempre poder suministrar una atención cordial, pues es imperativo como se indica en DCE estas personas:

"deben distinguirse por no limitarse a realizar con destreza lo más conveniente en cada momento, sino por su dedicación al otro con una atención que sale del corazón, para que el otro experimente su riqueza de humanidad. Por eso, dichos agentes, además de la preparación profesional, necesitan también y sobre todo una «formación del corazón» ... que suscite en ellos el amor y abra su espíritu al otro"48.

Al considerar la necesidad de apoyar a los otros también se puede considerar la propuesta que el Papa Emérito realiza cuando indica que "No puede dar únicamente y siempre, también debe recibir. Quien quiere dar amor, debe a su vez recibirlo como don" ${ }^{49}$. Allí ratifica que en estas actividades es necesario que consideremos la reciprocidad.

\section{Conclusiones}

En este estudio de las dos encíclicas ligadas con la caridad del Papa Emérito Benedicto XVI realizado con el apoyo de herramientas de la investigación cualitativa ha permitido identificar algunas claves que pueden ser de utilidad o apoyo para llevar a cabo actividades de proyección social, en especial cuando estas son adelantadas u orientadas por profesionales más ligados a las áreas técnicas. Las claves identificadas pueden ser empleadas tanto en las fases de formulación, diseño o ejecución de los proyectos e incluso podrían ser considerados más allá de los entornos ligados a la proyección social, en especial cuando se desea enriquecerlo con una mirada multidisciplinar y con un profundo sentido humano.

\footnotetext{
${ }^{47}$ DCE 31.

${ }^{48}$ DCE 31.

${ }^{49}$ DCE 7.
} 


\section{Bibliografía}

Benedicto XVI, Carta encíclica Caritas in veritate, $\mathrm{n}^{\circ} 1$ - 62, disponible en: http://www.vatican.va/content/benedict-xvi/es/encyclicals/documents/ hf_ben-xvi_enc_20090629_caritas-in-veritate.htmll, citado 27 enero 2021.

, Carta encíclica Deus caritas est, n 1-31, disponible en: http://www. vatican.va/content/benedict-xvi/es/encyclicals/documents/hf_ben-xvi_ enc_20051225_deus-caritas-est.html, citado 27 enero 2021.

, Fe y ciencia: un diálogo necesario, Sal Terrae, Santander 2011.

Cordes, J., "Deus caritas est. Sobre la primera encíclica del Papa Benedicto XVI", Scripta Theologica 38/3 (2006) 971-981.

CRoss, N., Engineering design methods: Strategies for product design, Wiley, New York 2020.

FARGNOLI, M.- SAKO, T., "Uncovering differences and similarities among quality function deployment-based methods in Design for X: Benchmarking in different domains", Journal Quality Engineering 29/4 (2017) 690-712.

Frémeaux, S.- Puyou, F. R. - Michelson, G., "Beyond accountants as technocrats: A common good perspective", Critical Perspectives on Accounting 67-68 (2020) 1- 14 .

GAÑán RoJo, P., "En el ejercicio de la caridad confluyen tecnología, ingeniería y humanismo", Revista Universidad Pontificia Bolivariana 58/158 (2019) $37-45$.

, "Responsabilidad individual y compromiso ambiental en los tiempos del COVID-19”, Revista Universidad Pontificia Bolivariana 59/159 (2020) 123-135.

García Melón, M. et al., Fundamentos del diseño en la ingeniería, Limusa, México 2010.

GLorioso, R., Introduction to engineering, Prentice Hall, New York 1974.

Gómez, V., La técnica: El umbral entre la cultura material y el materialismo de consumo. Por un debate social sobre la tecnociencia, Universidad Pontificia Bolivariana, Medellín 2020.

Iso, Guía DE RESPONSABILIDAD SOCIAL - ISO 26000:2010, Iso, Ginebra 2010, 1-5.

JAKOVLJEVIC, M., "Psychiatry and religion: Opponents or collaborators - The power of spirituality in contemporary psychiatry", Psychiatria Danubina 29/1 (2017) 82-88. 
Laurendeau, N., "Christian mysticism and science. The psychological dimension", Theology and Science 11/1 (2013) 8-31.

LuQue, D., "La educación del espíritu. Líneas de pensamiento para una teoría de la educación”, Edetania 57 (2020) 39-61.

Múnera, D., Curso de doctrina social de la Iglesia: humanismo cristiano y desarrollo humano, vol. 2, Industria Gráfica Litoservicios, Medellín 2012.

MuÑoz, R., “Caritas. Amor cristiano y acción social”, Scripta Theologica 38/3 (2006) 1005-1022.

Osses Bustingorry, S.- SÁnchez TAPiA, I.- Ibáñez Mansilla, F. M., "Inverstigación cualitativa en educación. Hacia la generación de teoría a través del proceso analítico", Estudios pedagógicos 32/1 (2006) 119-133.

Pablo VI, Carta Encíclica Populorum progressio, n. 2, disponible en: http:// www.vatican.va/content/paul-vi/es/encyclicals/documents/hf_p-vi_ enc_26031967_populorum.html, citado 27 enero 2021.

PÉREz BREVA, L., Innovating: a doer's manifesto for starting from a hunch, prototyping problems, scaling up, and learning to be productively wrong, The MIT Press, Cambridge 2018.

Pugh, S., Total design: integrated methods for successful product engineering, Prentice Hall, Harlow 1991.

RodríGuez, G.- Gil, J.- GARCíA, E., Metodología de la investigación cualitativa, Aljibe, Málaga 1996.

Salame, H. A., La planeación y el desarrollo de productos, Universidad Piloto de Colombia, Bogotá 2013.

SÁnchez Sorondo, M., Ex aedibvs academicis in civitate vaticana, Editrice Vaticana, Roma 2015.

SAPENA, J., et al., "Some reflections on poverty eradication, true development and sustainability within CST", Journal of Innovation \& Knowledge 3/2 (2018) 90-92.

Ulrich, K.- EpPInger, S., Product design and development, McGraw-Hill, Nueva York 2012.

Vaccaro, A.- Sison, A. J. G., "Transparency in business: The Perspective of Catholic Social Teaching and the "Caritas in Veritate", Journal of Business Ethics 100/1 (2011) 17-27. 